\title{
Years Ago in CORR
}

\section{Dupuytren's Contracture: A Guide for Management}

\author{
L. D. Howard MD CORR 1959;15:118-126
}

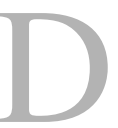

upuytren's disease remains a common clinical problem since its original description in 1834 [1]. In "Dupuytren's Contracture; a Guide for Management" published in CORR in 1959, L.D. Howard offered guidance in the operative treatment of this condition [3]. In spite of its age, many of the recommendations offered in the manuscript, especially with respect to surgical management, remain generally applicable today.

As most would agree, nonoperative treatment in the form of splinting remains "...poorly tolerated" and an "...attempt to lessen [the contracture] by splinting is simply unrealistic." Howard defines the criteria for nonoperative management as (1) mild, nondisabling contractures; (2) contractures in patients who cannot tolerate elective surgery; and (3) contractures in patients with substantial concomitant arthritis. Howard recorded limitation of extension and flexion by noting the distance from a line parallel to the metacarpals (Fig. 3) or the palm (Fig. 4), respectively.
Howard divided surgical treatment into three types. (1) Fasciotomy was recommended for contractures involving the metacarpophalangeal (MP) joint and not the proximal interphalangeal (PIP) joint due to the potential for iatrogenic nerve injury. The procedure is also advocated for patients who cannot tolerate more invasive surgery, those who cannot "...afford time off ...", and those prone to joint stiffness due to degenerative changes. (2) Limited fasciectomy was recommended for contractures with involvement within a

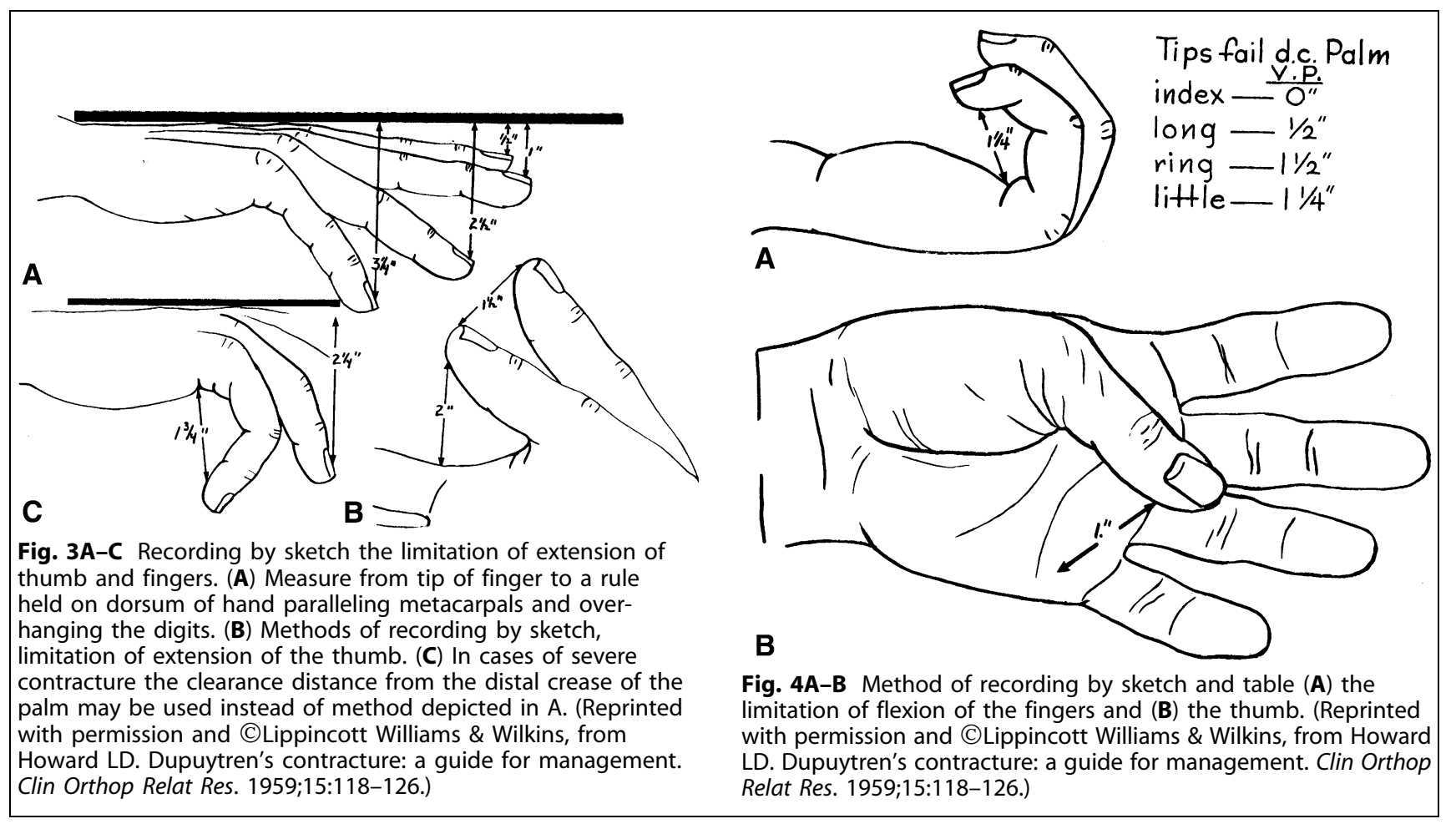


digit with the palm free of any involvement, those for older patients with longstanding contracture, and those prone to stiffness. (3) Radical fasciectomy was suggested for young patients with progressive disease and healthy individuals with "...widespread involvement and evidence of steady progression of the disease." These procedures and the respective indications remain by and large valid today, although there has been a trend toward the more limited procedures.
Many of the recommendations for intra and postoperative management are still in use. For example, the recommended surgical incisions and use of z-plasties to repair the skin are still in use today (Fig. 6). Other recommendations, including avoiding postoperative hematoma, the application of splints, early motion and the use of occupational therapy, are similarly applicable.

One area where the recommendations are out of step with today's conventional wisdom is in the area of

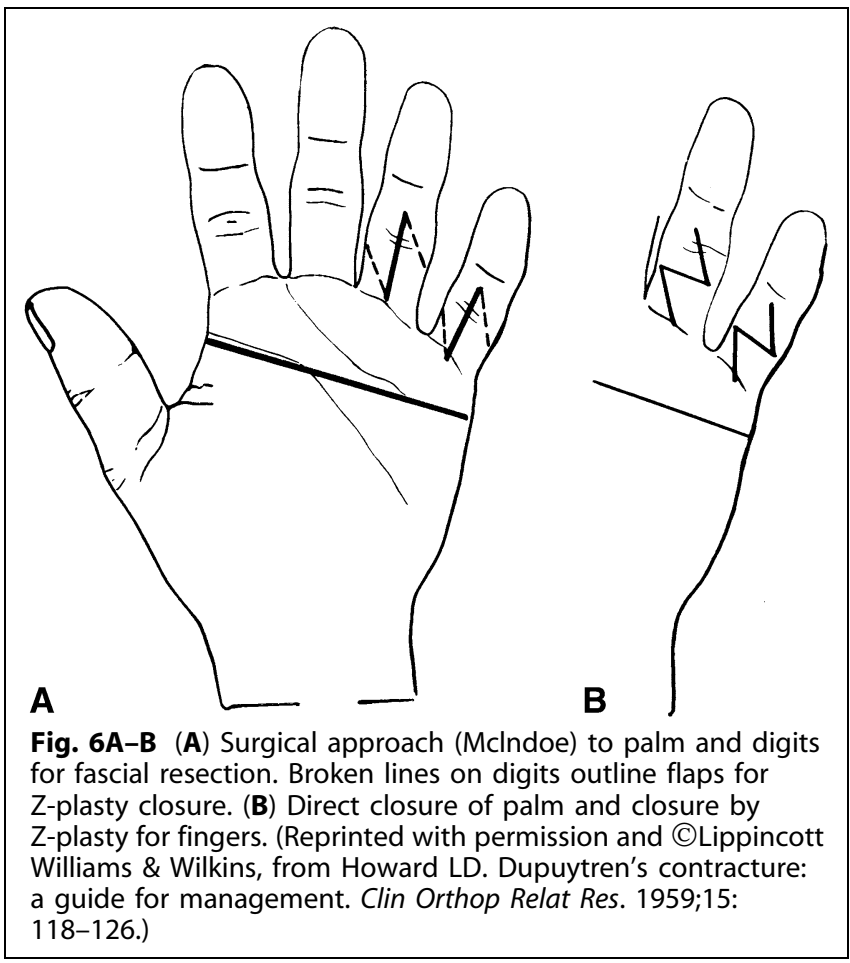

the management of open wounds. In this topic, Howard stated that, "It never pays to leave an open wound on the hand even for a short period of time." For patients with substantial skin involvement or contracture precluding primary wound closure, he recommended primary split or full thickness skin grafting. While grafting remains a useful tool in the surgeons' armamentarium in the management of soft tissues after Dupuytren's surgery, leaving some wounds open and allowing them to heal by secondary intention is an option for patients with severe disease [5].

Recently, two new approaches for management of Dupuytren's disease have been described. Needle aponeurotomy was popularized in France earlier in this decade as a minimally invasive approach to the treatment of this disease [2]. With this technique, a small gauge needle is used as a percutaneous cutting tool. The prominent cords are sectioned, and the affected digit is passively stretched to completely rupture the cord. While needle aponeurotomy has gained resurgent popularity among hand surgeons, the applicability of this technique for severe contractures, recurrence rate, and need for reoperation remain unclear.

Clostridial collagenase has recently been commercially introduced as a nonoperative alternative for the 


\section{Years Ago in CORR}

treatment of Dupuytren's disease [4]. This lytic enzyme is injected directly into the diseased tissue, and the affected digit is passively stretched one day after the injection to rupture the cord. Similar to NA, the use of collagenase for severe contractures (especially those involving the PIP joint), rate of recurrence rate, its cost effectiveness, and potential complications are not yet known.

The treatment of Dupuytren's disease has remained largely unchanged since Howard's work was published in1959. Surgical indications for the disease including progressive, debilitating contracture remain the same. Fasciotomy and limited fasciectomy continue to be the standard among treating surgeons. While NA and/or collagenase may represent the future for the management of the disease, more data and experience will be required to establish the relative effectiveness of these new technologies compared to traditional treatments.

Pedro K. Beredjiklian MD Chief, Division of Hand Surgery, The Rothman Institute, Philadelphia, PA

\section{References}

1. Dupuytren G. Permanent retraction of the fingers, produced by an affection of the palmar fascia. Lancet. 1834;2: 222-225.

2. Foucher G, Medina J, Navarro R. Percutaneous needle aponeurotomy: complications and results. J Hand Surg Br. 2003;28:427-431.
3. Howard LD. Dupuytren's contracture; a guide for management. Clin Orthop Relat Res. 1959;15:118-126.

4. Hurst LC, Badalamente MA, Hentz VR, Hotchkiss RN, Kaplan FT, Meals RA, Smith TM, Rodzvilla J; CORD I Study Group. Injectable collagenase clostridium histolyticum for Dupuytren's contracture. $N$ Engl J Med. 2009;361:968-979.

5. McCash CR. The open palm technique in Dupuytren's Contracture. $\mathrm{Br}$ J Plast Surg. 1964;17:271-280.

50 Years Ago in CORR:

Dupuytren's Contracture: A Guide

for Management

L. D. Howard MD

Pedro K. Beredjiklian MD 